\title{
ANALYSIS OF SOLUTES REJECTION IN ULTRAFILTRATION
}

\author{
SHIN-ICHI NAKAO AND SHOJI KIMURA \\ Institute of Industrial Science, University of Tokyo, \\ Tokyo 106
}

\begin{abstract}
To establish an effective method of quantitative analysis in ultrafiltration, the rejection of six solutions of various molecular weights by cellulose acetate ultrafiltration membranes was studied. At first, the effects of concentration polarization were corrected. Mass transfer coefficients determined by the velocity variation method agreed very well with the Deissler correlation. Next, by using Spiegler and Kedem equations, transport of solute through ultrafiltration membrane was analyzed. A method of curve-fitting was found effective to determine two parameters, i.e. solute permeability $P$ and reflection coefficient $\sigma$. Finally, these parameters obtained from experiments for various solutes were analyzed using the modified "pore theory", and were correlated with the ratio of the radius of solute and pore and with the effective pore length. Structure of the membrane was estimated from these results.
\end{abstract}

\section{Introduction}

With the development of practical applications of ultrafiltration in many industrial processes, analysis of both flux and rejection data has become increasingly important. However, until now rejection data have been analyzed less quantitatively than flux data.

For the quantitative analysis, it is necessary to correct concentration polarization, which is strong in ultrafiltration because of the smaller diffusivities of macromolecular solutes. This is achieved mathematically, using the concentration polarization model. It is also important to find transport equations which appropriately describe the permeation of water and solutes through a membrane, but as yet this has not been accomplished.

The objective of this study is to establish an analytical method of ultrafiltration rejection data. For this purpose, concentration polarization is first corrected, using the concentration polarization model and a method of velocity variation. Secondly, transport equations are determined based on nonequilibrium thermodynamics. As these equations contain three parameters which specify rejection characteristics of a membrane, a method of determining these parameters is investigated. Last, the parameters obtained are discussed, using the "pore theory".

\section{Theoretical}

\section{1 Correction of concentration polarization}

The rejection characteristics of a membrane are

Received april 4, $1980 . \quad$ Correspondence concerning this article should be addressed to S. Nakao. usually described by the observed rejection $R_{\mathrm{obs}}$, defined as

$$
R_{\mathrm{obs}}=\left(C_{b}-C_{p}\right) / C_{b}
$$

where $C_{b}$ and $C_{p}$ denote bulk and permeate concentration, respectively. In membrane separation processes, however, a concentration at the membrane surface $C_{m}$ is always higher than that in the bulk $C_{b}$ because of the concentration polarization phenomenon. Corrected rejection characteristics of the membrane, therefore, are described by the real rejection $R$, defined as

$$
R=\left(C_{m}-C_{p}\right) / C_{m}
$$

The value of $C_{m}$ is not directly obtainable by experiment, so it must be calculated using the following equation based on the concentration polarization mode $^{6 !}$.

$$
J_{v}=k \cdot \ln \left\{\left(C_{m}-C_{p}\right) /\left(C_{b}-C_{p}\right)\right\}
$$

According to this equation, if the value of $k$, which is the mass transfer coefficient in the boundary layer, is known, we can calculate the value of $C_{m}$.

The velocity variation method is used to determine the mass transfer coefficient ${ }^{3,7)}$. The value of $k$ is usually a function of the Reynolds number and can be given as

$$
k \propto u^{a}
$$

Using this relation, Eq. (3) is rewritten as

$$
\ln \left(\begin{array}{c}
1-R_{\mathrm{obs}} \\
R_{\mathrm{obs}}
\end{array}\right)=\ln \left(\frac{1-R}{R}\right)+b\left(\frac{J_{v}}{u^{a}}\right)
$$

This means that the linear plot of $\ln \left(1-R_{\mathrm{obs}}\right) / R_{\mathrm{obs}}$ vs. $J_{v} / u^{a}$ is obtained and the true rejection $R$ is given by extrapolation to an ordinate axis. Using this $R$, 
the mass transfer coefficient is calculated by Eq. (3), and the effects of concentration polarization can be corrected.

\subsection{Transport equations}

Various transport equations for reverse osmosis and ultrafiltration membranes have been presented by different authors.

Some of them were developed on the basis of a particular transport mechanism. For example, Jonsson and Boesen ${ }^{2}$ proposed a transport equation by adopting the finely-porous model and applied their equation to the analysis of reverse osmosis and ultrafiltration data.

The other way is to consider a membrane as a "black box", and the transport equations are determined phenomenologically on the basis of nonequilibrium thermodynamics. As the permeation mechanism of ultrafiltration membrane is not clear at this stage, the "black box" treatment seems more reasonable than the assumption of particular mechanisms.

Kedem and Katchalsky ${ }^{4}$ derived the following equations for permeating through a membrane using nonequilibrium thermodynamics.

$$
\begin{gathered}
J_{v}=L_{p}(\Delta P-\sigma \Delta \Pi) \\
J_{s}=P\left(C_{m}-C_{p}\right)+(1-\sigma) J_{v} \bar{C}
\end{gathered}
$$

In these equations, the membrane characteristics are described by three parameters: pure water permeability $L_{p}$, solute permeability $P$ and reflection coefficient $\sigma$. The determination of these parameters is very important. The term $\bar{C}$ in Eq. (7) shows the average concentration on both sides of a membrane, and the logarithmic mean is usually used.

In the case of a high-rejection ultrafiltration system, the value of $\Delta C$ is so large that the logarithmic mean concentration no longer corresponds to the correct average. To solve this problem, Spiegler and Kedem ${ }^{11}$ divided the membrane into differential elements in the direction of its thickness and applied Eq. (7) in it as the form of differential equation. The result of integration is expressed as

$$
R=\sigma(1-F) /(1-\sigma F)
$$

where

$$
F=\exp \left\{-(1-\sigma) \cdot J_{v} / P\right\}
$$

Equation (6) does not change by this treatment.

These equations have already been used as transport equations of a reverse osmosis membrane (only in the case of highly rejected solute), but for an ultrafiltration membrane their application has never been reported. So it is very important to determine whether these equations are appropriate for ultrafiltration in all

\begin{tabular}{|c|c|c|c|}
\hline Solute & Molecular weight & $D \times 10^{8}\left[\mathrm{~cm}^{2} / \mathrm{s}\right]$ & $r_{s} \times 10^{8}[\mathrm{~cm}]$ \\
\hline PEG \#4000 & 3000 & 1.5 & 16.3 \\
\hline Vitamin $B_{12}$ & 1355 & 3.3 & 7.4 \\
\hline Raffinose & 504 & 4.2 & 5.8 \\
\hline Sucrose & 342 & 5.2 & 4.7 \\
\hline Glucose & 180 & 6.9 & 3.6 \\
\hline Glycerin & 92 & 9.5 & 2.6 \\
\hline
\end{tabular}
regions of rejection. At the same time, the determination method of parameters used in these equations is also very important. These two objects are the main part of this study.

\section{Experimental}

\section{1 Apparatus and materials}

Two kinds of cellulose acetate ultrafiltration tubular membranes (T2/A and T4/A) produced by Paterson Candy International, Ltd., England, were employed in this study. The flux through these membranes is not so large that plugging by macromolecular solutes does not occur, and their rejection abilities are very stable.

The experimental apparatus of the "system 1" explained in the authors' previous study ${ }^{8}$ ' was used in this work.

Six kinds of solutes were used: polyethylene glycol (PEG 4000 ), vitamin $B_{12}$, raffinose, sucrose, glucose, and glycerin. Molecular weights, diffusivities and molecular radii of these solutes are shown in Table 1. Diffusivity of PEG $\$ 4000$ was measured by the ultracentrifugal method and other values were taken from the literature ${ }^{10,13,14)}$ and corrected for experimental temperature. Molecular radius $r_{s}$ was calculated by following the Stokes-Einstein equation.

$$
r_{s}=\mathscr{K} T / 6 \Pi \mu D
$$

As the solutions used in the experiment were dilute, the viscosity of water, 0.0089 poise, was used in the above calculations.

\section{2 Conditions and procedure}

Two kinds of experiments were performed. In one kind of experiment, four feed velocities $(38,58,100$, $140 \mathrm{~cm} / \mathrm{s}$ ) were employed and the pressure was kept constant at $8 \mathrm{~atm}$. In the other, various pressures between 2 and $12 \mathrm{~atm}$ were employed and the velocity was kept constant at $58 \mathrm{~cm} / \mathrm{s}$. The temperature of feed solution was controlled at $25^{\circ} \mathrm{C}$ by a thermostat throughout all the experimental runs. The concentration of the feed solution ranged from 100 to 2000 ppm.

The concentrations of vitamin $B_{12}$ were analyzed spectrophotometrically at $361 \mathrm{~nm}$ and other solute concentrations were measured using a total organic carbon analyzer.

The experimental procedure was as follows. Pure water permeability was measured at the start of each experiment. Then, the feed water was changed to 


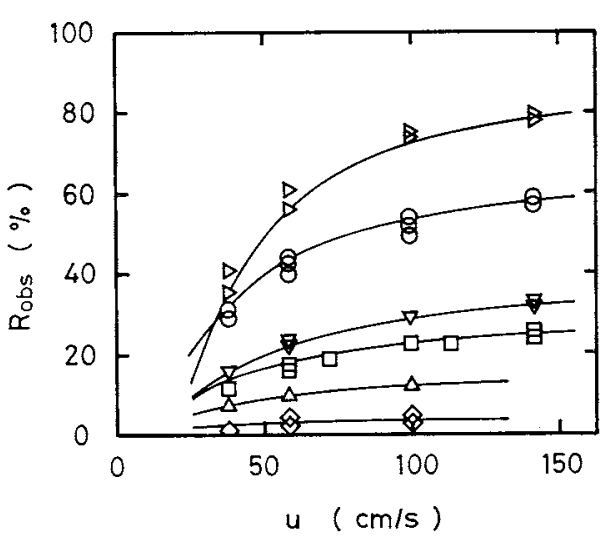

$\triangleright$ PEG $\$ 4000 \circ$ Vitamin $B_{12} \nabla$ Raffinose

$\square$ Sucrose $\triangle$ Glucose $\diamond$ Glycerin

Keys are the same in all figures.

Fig. 1 Effect of feed velocity on observed rejection of T4/A membrane at $8 \mathrm{~atm}$

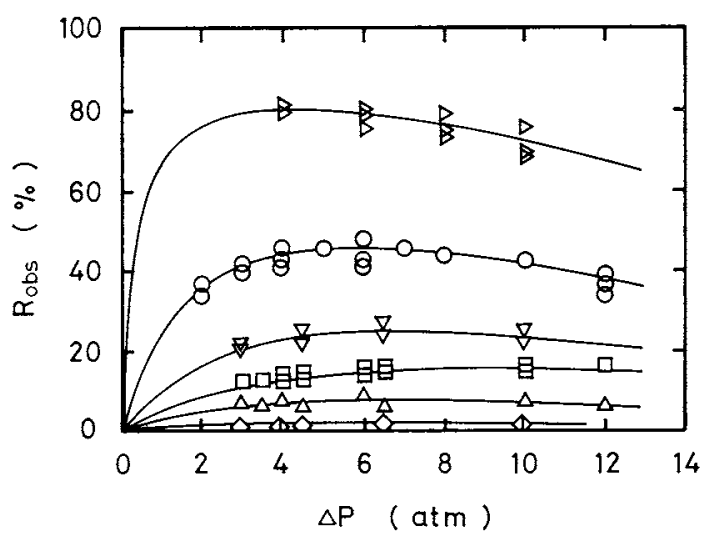

Fig. 2 Effect of pressure on observed rejection of T4/A membrane at $58 \mathrm{~cm} / \mathrm{s}(100 \mathrm{~cm} / \mathrm{s}$ only for PEG\#4000)

the experimental solution. The permeate flux and concentration were measured after about $30 \mathrm{~min}$, when these values reached steady state. Then the flow rate or pressure was changed to the next value.

\section{Results and Discussion}

The pure water permeability was almost constant throughout the experiments, thus the characteristics of the membranes could be kept constant. Also, the volume fluxes of various experiments were the same as those for the pure water. This shows that the effect of osmotic pressure was negligible and that plugging or gel layer formation did not occur.

\section{1 Effects of feed velocity and pressure}

Effects of feed velocity and pressure on the observed rejection are shown in Figs. 1 and 2. In Fig. 1, $R_{\mathrm{obs}}$ of high rejected solute changed considerably with feed velocity. The effect of pressure was smaller than that of feed velocity as shown in Fig. 2 and $R_{\mathrm{obs}}$ decreased with increasing pressure. All these effects were caused by the concentration polarization and its correction

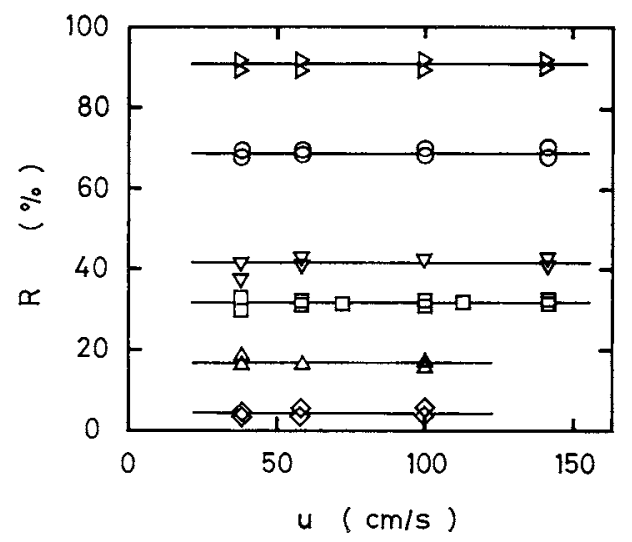

Fig. 3 Effect of feed velocity on real rejection of T4/A membrane at $8 \mathrm{~atm}$

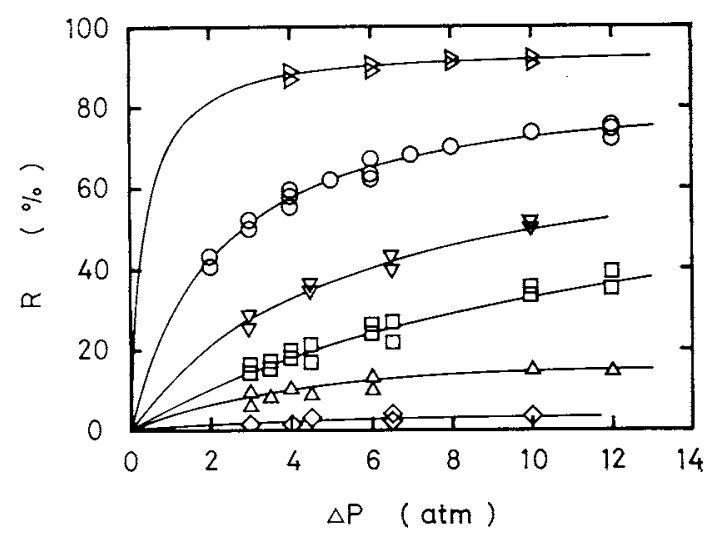

Fig. 4 Effect of pressure on real rejection of T4/A membrane at $58 \mathrm{~cm} / \mathrm{s}(100 \mathrm{~cm} / \mathrm{s}$ only for PEG 4000$)$

by Eq. (3) was very important. The real rejection $R$ is shown in Figs. 3 and 4 . There was no longer an effect of feed velocity, as shown in Fig. 3. But the effect of pressure remained, as shown in Fig. 4, and the value of $R$ increased with pressure. Thus $R$ is not a proper parameter to express the characteristics of a membrane.

These four figures also show that there was no effect of concentration on $R_{\mathrm{obs}}$ or $R$ in this concentration range.

The lines in these figures exhibit theoretical values calculated by means of Eqs. (1), (3), (6), (8), (9) and (11) and the values of $\sigma$ and $P$ determined by curve fitting, which will be discussed later.

\section{2 Mass transfer coefficient}

As mentioned above, mass transfer coefficient was measured by the method of velocity variation. The plots of $\ln \left\{\left(1-R_{\mathrm{obs}}\right) /\left(R_{\mathrm{obs}}\right\}\right.$ vs. $J_{v} / u^{0.875}$ gave nearly perfect straight lines. The power of 0.875 was chosen on the basis of the following Deissler equation which was used in our previous study ${ }^{8}$.

$$
N_{S h}=0.023 \cdot N_{R e^{0.875}} N_{S e^{0.25}}
$$

The comparison between measured and calculated values of mass transfer coefficient using Eq. (11) is 


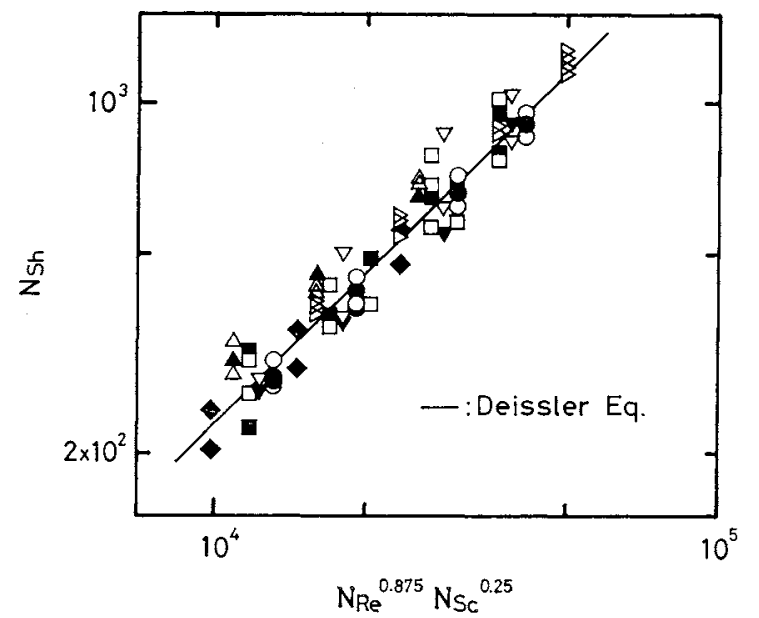

White and black keys indicate T4/A and T2/A membranes respectively.

Fig. 5 Comparison between experimental and calculated mass transfer coefficient values using Deissler correlation

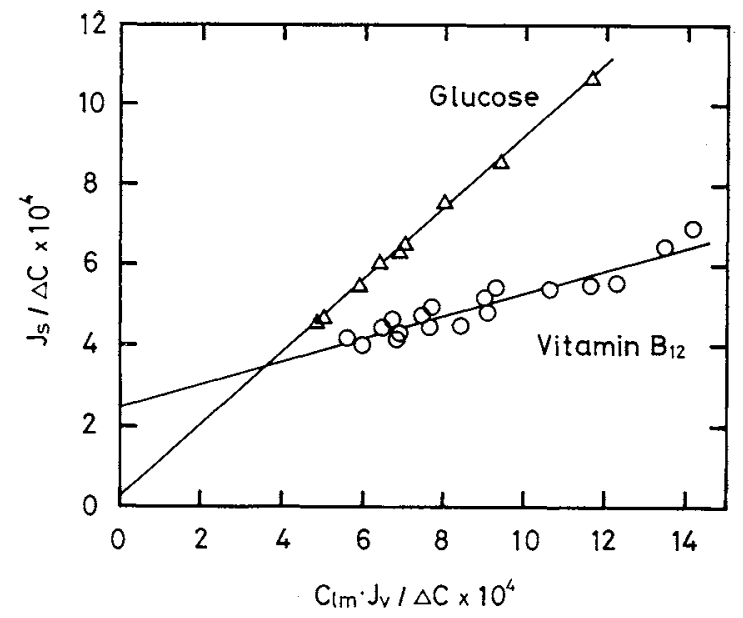

Fig. 6 Determination of $\sigma$ and $P$ of $\mathrm{T} 4 / \mathrm{A}$ membrane by using logarithmic mean concentration

illustrated in Fig. 5, in which diffusivities in Table 1 and water viscosity, 0.0089 poise, are used. Both values agreed very well for all solutes and membranes, showing that the Deissler correlation was appropriate to estimate mass transfer coefficients.

\section{3 Determinations of membrane parameters}

Three parameters, $L_{p}, \sigma$ and $P$, which describe the membrane characteristics were determined in the following manner.

Pure water permeability $L_{p}$ was obtained from the experiment of pure water permeation using Eq. (6), in which the osmotic pressure difference $\Delta I I$ is zero.

Two parameters $\sigma$ and $P$ were first determined by using the logarithmic mean concentration $C_{l m}$. Equation (7) is rewritten as

$$
J_{s} / \Delta C=P+(1-\sigma) \cdot\left(J_{v} C_{l m} / \Delta C\right)
$$

where $\Delta C=C_{m}-C_{p}$ is the concentration difference. As illustrated in Fig. 6, there is a good linear correlation between $J_{s} / \Delta C$ and $J_{v} \cdot C_{l m} / \Delta C$, and the parameters

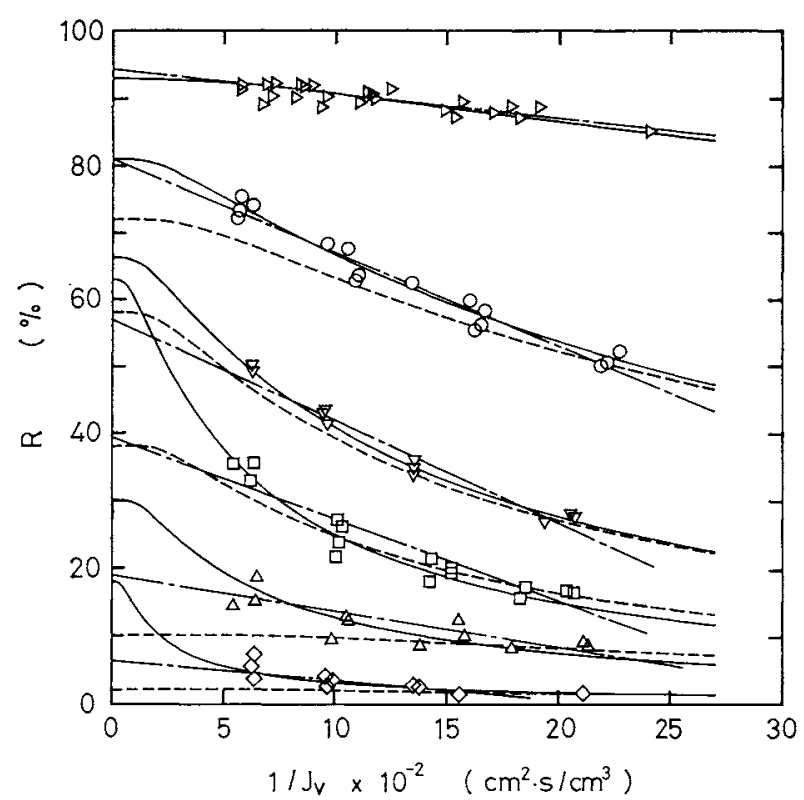

_-_.: determined by the method of curve fitting

-.-.-: determined by using logarithmic mean concentration

- - : extrapolation of $1 / J_{v} \rightarrow 0$ for determination of $\sigma$

Fig. 7 Plots of $R$ vs. $1 / J_{v}$

$\sigma$ and $P$ were obtained from the slope and intercept. Then, to examine the accuracy of these parameters, the real rejection $R$ was calculated by means of Eqs. (8) and (9) using these values, and plotted versus $1 / J_{v}$ as dotted lines in Fig. 7. In the region of low flux, the calculated lines agreed well with the experimental data, but in the high-flux area, the agreement between the two was not good. The use of logarithmic mean concentration, therefore, is only valid in the low-flux region.

In Eqs. (8) and (9), $F \rightarrow 0$ at high flux and hence the reflection coefficient $\sigma$ is the limiting value of $R$.

$$
J_{v} \rightarrow \infty ; R \rightarrow \sigma
$$

This fact has been used for the determination of $\sigma$, by plotting $R$ vs. $J_{v}$ or $R$ vs. $1 / J_{v}$ and by extrapolating $J_{v} \rightarrow \infty$ or $1 / J_{v} \rightarrow 0$. But it was only in the case of high or low rejected solutes that this plot became a straight line, as illustrated in Fig. 7, and the values of $\sigma$ extrapolated were not accurate.

Direct curve-fitting of Eqs. (8) and (9) was also attempted. When $\sigma$ is assumed to have a certain value, the average and standard deviation of $P$ for all experimental data of one solute and membrane can be calculated using Eqs. (8) and (9). By calculating the standard deviation at a different value of $\sigma$, its most suitable value is determined when the standard deviation becomes minimum. $P$ is then given as the average of all data. The best fitting curves are illustrated in Fig. 7 as solid lines and the values of $\sigma, P$ and its standard deviation are listed in Table 2. The calculated lines agreed very well with the experimental data for all solutes. 


\begin{tabular}{|c|c|c|c|}
\hline Solute & $\sigma$ & $\begin{array}{l}P \times 10^{4} \\
{[\mathrm{~cm} / \mathrm{s}]}\end{array}$ & $\begin{array}{l}\text { Standard } \\
\text { deviation }\end{array}$ \\
\hline PEG $\# 4000$ & 0.93 & 0.52 & $1.7 \times 10^{-5}$ \\
\hline Vitamin $\mathbf{B}_{12}$ & 0.81 & 3.0 & $3.1 \times 10^{-5}$ \\
\hline Raffinose & 0.66 & 7.8 & $3.9 \times 10^{-5}$ \\
\hline Sucrose & 0.63 & 17 & $1.8 \times 10^{-4}$ \\
\hline Glucose & 0.30 & 17 & $4.5 \times 10^{-4}$ \\
\hline Glycerin & 0.18 & 55 & $1.7 \times 10^{-3}$ \\
\hline
\end{tabular}

Table 3 Pore radii and the values of $A_{k} / \Delta X$ of the T4/A membrane

\begin{tabular}{lcc}
\multicolumn{1}{c}{ Solute } & $\begin{array}{c}r_{p} \times 10^{8} \\
{[\mathrm{~cm}]}\end{array}$ & $\begin{array}{c}A_{k} / \Delta X \\
{\left[\mathrm{~cm}^{-1}\right]}\end{array}$ \\
\hline PEG $\# 4000$ & 21.2 & - \\
Vitamin $\mathrm{B}_{12}$ & 11.6 & 11000 \\
Raffinose & 11.2 & 4800 \\
Sucrose & 9.4 & 3500 \\
Glucose & 11.8 & 1240 \\
Glycerin & 11.7 & 1730
\end{tabular}

Average $r_{p}$ (excluding PEG $\$ 4000$ and sucrose) $=11.6 \times 10^{-8}$ $A_{k} / \Delta X$ obtained from pure water permeation $=875 \mathrm{~cm}^{-1}$

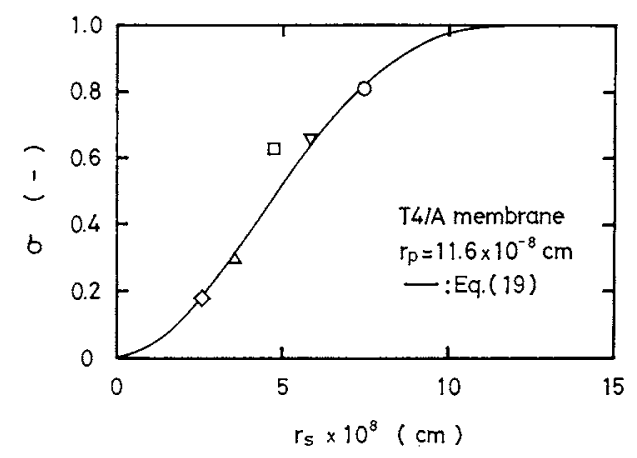

Fig. 8 Relationship between reflection coefficient and the Stokes radius of solute for T4/A membrane

It is apparent from all these results that the Spiegler and Kedem equations, Eqs. (8) and (9), were suitable as transport equations of ultrafiltration and that the curve-fitting method was the best for the determination of the membrane parameters, $\sigma$ and $P$. Furthermore, these equations and parameters could aptly explain unanalyzed data as illustrated in Figs. 1-4.

\section{4 The "pore theory"}

Pappenheimer et al. ${ }^{9 \prime}$ proposed the "pore theory" to account for transcapillary transport. In this theory, the solute flux consists of filtration and diffusion flow and both these flows are impeded by steric hindrance at the entrance of the membrane pore and by frictional forces within the pores.

Verniory et al. ${ }^{12)}$ modified this "pore theory", adopting Haberman and Sayre's correction ${ }^{13}$ of the frictional forces and relating it to Kedem and Katchalsky's treatment ${ }^{5}$ based on nonequilibrium thermodynamics. This modified "pore theory" was applied to the analysis of hemodialysis by Wendt et al. ${ }^{14}$. According to this theory, the membrane structure can be estimated by the parameters $\sigma$ and $P$. In our study, an attempt was made to find the structural implications of the ultrafiltration membrane using this "pore theory".

Assuming that the cylindrical membrane pore has a constant radius $r_{p}$ and length $\Delta X$ and that the spherical solutes have a radius $r_{s}$, Verniory et al. describe the solute flux $J_{s}$ as

$$
J_{s}=D \cdot f(q) S_{D} \cdot \frac{A_{k}}{\Delta X} \cdot\left(C_{m}-C_{p}\right)+J_{v} \cdot C \cdot g(q) \cdot S_{W^{\prime}}
$$

where $A_{k}$ is the ratio of the total cross-sectional pore area to the effective membrane area. $S_{D}$ and $S_{F}$ are the steric hindrance factors for diffusion and filtration flow respectively and are functions of $q$, which is the ratio of $r_{s}$ to $r_{p}$. They are defined as

$$
\begin{gathered}
S_{D}=(1-q)^{2} \\
S_{F}=2(1-q)^{2}-(1-q)^{4}
\end{gathered}
$$

$f(q)$ and $g(q)$ are the correction factors for the effects of a cylinder wall, and were calculated by Haberman and Sayre as follows.

$$
\begin{gathered}
f(q)=\left(1-2.1 q+2.1 q^{3}-1.7 q^{5}+0.73 q^{6}\right) /\left(1-0.76 q^{5}\right) \\
g(q)=\left\{1-(2 / 3) q^{2}-0.2 q^{5}\right\} /\left(1-0.76 q^{5}\right)
\end{gathered}
$$

Comparing Eq. (14) with Eq. (7), the membrane parameters $\sigma$ and $P$ are expressed as

$$
\begin{gathered}
\sigma=1-g(q) \cdot S_{F} \\
P=D \cdot f(q) \cdot S_{D} \cdot\left(A_{k} / \Delta X\right)
\end{gathered}
$$

1) Effect of pore radius Assuming that the ultrafiltration membrane pore is a cylindrical tube of a constant radius $r_{p}$, its value can be calculated by Eq. (19) with the solute radius and $\sigma$ listed in Tables 1 and 2, and the results are given in Table 3. Except for PEG $\$ 4000$ and sucrose, the values of $r_{p}$ were in good agreement and the average value, $r_{p}=11.6 \times$ $10^{-8} \mathrm{~cm}$, was obtained. In the case of PEG $\$ 4000$, the use of the Stokes-Einstein formula for the calculation of $r_{s}$ is not appropriate because it is a linear chain polymer. The relationship between the reflection coefficient $\sigma$ and the solute radius $r_{s}$ is illustrated in Fig. 8. The theoretical line calculated by Eq. (19) agreed well with the experimental data.

The effects of a distribution of pore diameters were studied. If there is a Gaussian distribution in the pore radii, the value of $r_{p}$ based upon the assumption of uniform radius is the largest of all mean radii obtained with the distribution. The line calculated with the constant $r_{p}$ fitted best to the experimental data, and the curve based upon the Gaussian distribution became more gently-sloping. Thus, one can infer that there is little distribution of pore diameter in this ultrafiltration membrane.

2) Effect of pore length The pore length $\Delta X$ cannot be calculated, but the value of $A_{k} / \Delta X$ can be determined using Eq. (20) and the value $r_{p}$. As $A_{k}$ is constant for a certain membrane, this value can be 
considered to exhibit the length $\Delta X . A_{k} / \Delta X$ is also given by the pure water flux $J_{w}$ using the HagenPoiseuille equation as

$$
J_{w}=\left(r_{p}^{2} / 8 \mu\right) \cdot\left(A_{k} / \Delta X\right) \cdot \Delta P
$$

If the value of $r_{p}$ is known, $A_{k} / \Delta X$ can be calculated.

Results of these calculations using $r_{p}=11.6 \times 10^{-8}$ $\mathrm{cm}$ are also listed in Table 3. If the membrane consists of cylindrical pores which are not connected to each other, it follows that the value of $A_{k l} / \Delta X$ is constant for the same membrane. The results shown in Table 3, however, increased with solute radius $r_{s}$. As illustrated in Fig. 9, $A_{k} / \Delta X$ decreased in proportion to $r_{s}^{3}$ and became constant at the value determined by Eq. (21). These results mean that the pore length $\Delta X$ is the same value as for pure water in the small solute region and that it decreases in proportion to $r_{s}^{3}$ above a certain radius particular for that membrane. From these results, one can visualize the following structure of membrane pores and mechanism of solute permeation. This cellulose acetate ultrafiltration membrane consists of cylindrical pores which are connected to one another. Large solutes cannot pass through these connected points and go straight, but small ones and water can pass through freely, so that the values of $\Delta X$ vary with the solute. The difficulty of passing through at this point is assumed to depend on the volume of a solute; in other words, $A_{k} / \Delta X$ is in proportion to $r_{s}^{3}$. Further research is now underway to determine the applicability of this concept to other types of membranes.

\section{Conclusion}

Mass transfer coefficients necessary to correct the concentration polarization were determined by the method of velocity variation and agreed very well with the Deissler correlation.

Equations (6), (8) and (9) were applicable as transport equations of ultrafiltration and the membrane parameters $\sigma$ and $P$ were obtained by the method of curve-fitting. Other methods seemed to be less suitable than the above method.

The parameters $\sigma$ and $P$ could be explained by the "pore theory" modified by Verniory et al. According to this theory, the pore radius and length of the membrane were estimated from the values of $\sigma$ and $P$, and it became clear that the pore length was in inverse proportion to the 3 rd power of the solute radius.

\section{Nomenclature}

$\begin{array}{llr}A_{k} & =\text { ratio of total cross-sectional pore area to } \\ & \text { effective membrane area } \\ C & =\text { concentration of solute } & {[-]} \\ D & =\text { diffusivity } & {\left[\mathrm{mol}^{2} \mathrm{~cm}^{2}\right]} \\ F & =\text { quantity defined by Eq. }(10) & {\left[\mathrm{cm}^{2} / \mathrm{s}\right]} \\ f(q), g(q) & =\text { wall correction factors for diffusion and } \\ & \text { filtration flow, respectively }\end{array}$

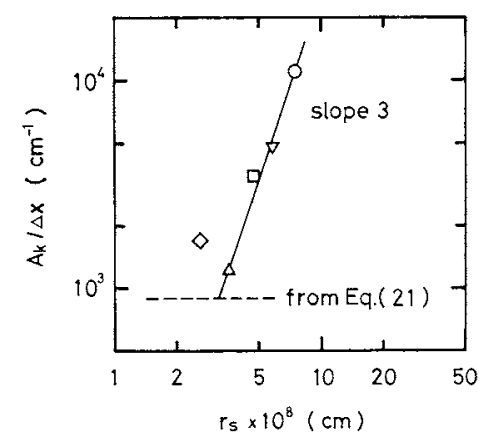

Fig. 9 Relationship between $A_{k} / \Delta X$ and Stokes radius of solute for $\mathrm{T} 4 / \mathrm{A}$ membrane

\begin{tabular}{|c|c|c|}
\hline$J_{s}$ & $=$ solute flux through membrane & {$\left[\mathrm{mol} / \mathrm{cm}^{2} \cdot \mathrm{s}\right]$} \\
\hline$J_{v}$ & $=$ volume flux through membrane & {$\left[\mathrm{cm}^{3} / \mathrm{cm}^{2} \cdot \mathrm{s}\right]$} \\
\hline$J_{w}$ & $=$ volume flux of pure water & {$\left[\mathrm{cm}^{3} / \mathrm{cm}^{2} \cdot \mathrm{s}\right]$} \\
\hline$k$ & $=$ mass transfer coefficient & {$[\mathrm{cm} / \mathrm{s}]$} \\
\hline $\mathscr{R}$ & $=$ Boltzmann's constant & [erg/deg] \\
\hline$L_{p}$ & $=$ pure water permeability & {$\left[\mathrm{cm}^{3} / \mathrm{cm}^{2} \cdot \mathrm{s} \cdot \mathrm{atm}\right]$} \\
\hline$N_{R e}$ & $=$ Reynolds number & {$[-]$} \\
\hline$N_{S c}$ & $=$ Schmidt number & {$[-]$} \\
\hline$N_{S h}$ & $=$ Sherwood number & {$[-]$} \\
\hline$P$ & $=$ solute permeability & {$[\mathrm{cm} / \mathrm{s}]$} \\
\hline$\Delta P$ & $=$ pressure difference & [atm] \\
\hline$q$ & $=r_{s} / r_{p}$ & {$[-]$} \\
\hline$R$ & $=$ real rejection & {$[-]$} \\
\hline$R_{\mathrm{obs}}$ & $==$ observed rejection & {$[-]$} \\
\hline$r_{p}$ & $=$ radius of membrane pore & [cm] \\
\hline & $=$ Stokes radius of solute & [cm] \\
\hline$S_{D}, S_{F}$ & $\begin{aligned}= & \text { steric hindrance factors for diffus } \\
& \text { filtration flow, respectively }\end{aligned}$ & ision and \\
\hline$T$ & $=$ absolute temperature & [K] \\
\hline$u$ & $=$ feed velocity & {$[\mathrm{cm} / \mathrm{s}]$} \\
\hline$\Delta X$ & $=$ pore length & [cm] \\
\hline$\mu$ & $=$ viscosity & {$[\mathrm{g} / \mathrm{cm} \cdot \mathrm{s}]$} \\
\hline$\Delta I I$ & $=$ osmotic pressure difference & [atm] \\
\hline$\sigma$ & $=$ reflection coefflcient & [-] \\
\hline
\end{tabular}

\section{Literature Cited}

1) Haberman, W. L. and R. M. Sayre: David Taylor Model Basin Report No. 1143, Department of the Navy (1958).

2) Jonsson, G. and C. E. Boesen: Desalination, 17, 145 (1975).

3) idem: ibid., 21, 1 (1977).

4) Kedem, O. and A. Katchalsky: Biochim. Biophys. Acta, 27, 229 (1958).

5) idem: J. Gen. Physiol., 45, 143 (1961).

6) Kimura, S. and S. Sourirajan: AIChE J., 13, 497 (1967).

7) Kimura, S.: Bull. Soc. Sea Water Sci. Jpn., 27, 295 (1974).

8) Nakao, S., T. Nomura and S. Kimura: AIChE J., 25, 615 (1979).

9) Pappenheimer, J. R., E. M. Renkin and L. M. Borrero: Am. J. Physiol., 167, 13 (1951).

10) Sourirajan, S.: "Reverse Osmosis", Logos Press, London (1970).

11) Spiegler, K. S. and O. Kedem: Desalination, 1, 311 (1966).

12) Verniory, A., R. Du Bois, P. Decoodt, J. P. Gassee and P. P. Lambert: J. Gen. Physiol., 62, 489 (1973).

13) Washburn, E. W., ed.: "International Critical Tables", Vol. V, McGraw-Hill, N. Y. (1929).

14) Wendt, R. P., E. Klein, E. H. Bresler, F. F. Holland, R. M. Serino and H. Villa: J. Membrane Sci., 5, 23 (1979). 